\title{
Comparison of two attenuated infectious bursal disease vaccine strains focused on safety and antibody response in commercial broilers
}

\author{
Thotsapol Thomrongsuwannakij ${ }^{1}$ (D), Nataya Charoenvisal ${ }^{2}$ (D) and Niwat Chansiripornchai² \\ 1. Akkhraratchakumari Veterinary College, Walailak University, Nakorn Si Thammarat 80160, Thailand; 2. Avian Health \\ Research Unit, Department of Veterinary Medicine, Faculty of Veterinary Science, Chulalongkorn University, \\ Bangkok 10330, Thailand. \\ Corresponding author: Niwat Chansiripornchai, e-mail: niwat.c@chula.ac.th \\ Co-authors: TT: thotsapol.th@wu.ac.th, NC: nataya.c@chula.ac.th \\ Received: 18-08-2020, Accepted: 23-11-2020, Published online: 11-01-2021
}

doi: www.doi.org/10.14202/vetworld.2021.70-77 How to cite this article: Thomrongsuwannakij T, Charoenvisal N, Chansiripornchai N (2021) Comparison of two attenuated infectious bursal disease vaccine strains focused on safety and antibody response in commercial broilers, Veterinary World, 14(1): 70-77.

\begin{abstract}
Background and Aim: Infectious bursal disease (IBD) or Gumboro disease is one of the most detrimental diseases in the poultry industry worldwide. Previous scientific studies have shown that live IBD vaccination might induce transient immunosuppression, leading to suboptimal vaccine responses, and therefore lack of protection against other infectious diseases; therefore, selecting an IBD vaccine in commercial farms is a concern. This study aims to compare two commercially attenuated IBD vaccines (intermediate and intermediate-plus strains) in terms of safety and antibody response to IBD and Newcastle disease viruses (NDV) in commercial broilers.

Materials and Methods: Overall, 216 Cobb broiler chickens were divided into three groups based on the IBD vaccine strain administered: V217 strain (Group 1), M.B. strain (Group 2), and an unvaccinated group (Group 3). Groups 1 and 2 were orally vaccinated with Hitchner B1 NDV vaccine strain 7 days after IBD vaccination. Blood samples were collected at IBD vaccination day (15 days of age) and at $7,14,21$, and 28 days post-IBD vaccination. The immunosuppressive effects of the IBD vaccination were determined by NDV antibody response, the bursa:body weight (B:BW) ratio, and the histopathological lesion scores of the bursa of Fabricius. Phylogenetic analysis was also performed.

Results: Phylogenetic analysis revealed that the M.B. strain belonged to a very virulent IBD strain, whereas the V217 strain belonged to a classical IBD virus strain. NDV antibody titers of the two vaccinated groups increased after ND vaccination, reaching their maximum at 14 days post-ND vaccination and decreasing thereafter. The V217 group presented the highest NDV humoral response from 7 days post-vaccination (dpv) to the end of the study. The mean NDV antibody titer of the V217 group was significantly $(\mathrm{p}<0.05)$ higher than that of the M.B. group at $14 \mathrm{dpv}$. In addition, the V217 strain-induced lower bursal lesions post-IBD vaccination and a higher B:BW ratio at 7 and $21 \mathrm{dpv}$ compared to the M.B. group. The higher B:BW ratio, lower bursal lesions, and higher ND antibody response present in the V217 group indicate that the V217 strain induces lower immunosuppressive effects compared to the M.B. strain.
\end{abstract}

Conclusion: The results of this study indicate that IBD vaccine selection merits consideration, as avoiding the immunosuppressive effects induced by live IBD vaccination and the consequent impact on response to other vaccines is important.

Keywords: broilers, immunosuppressive effects, infectious bursal disease, vaccination.

\section{Introduction}

Infectious bursal disease (IBD), also known as Gumboro disease, is one of the most widespread immunosuppressive avian diseases, causing high morbidity and mortality in commercial broilers, and even up to $100 \%$ mortality in susceptible white leghorn chickens $[1,2]$. This disease is caused by the IBD virus (IBDV), which is a single-shelled, non-enveloped virus with a double-stranded ribonucleic acid (RNA) genome [2,3]. This virus is considerably resistant to harsh environments due to the absence of an envelope.

Copyright: Thomrongsuwannakij, et al. Open Access. This article is distributed under the terms of the Creative Commons Attribution 4.0 International License (http://creativecommons. org/licenses/by/4.0/), which permits unrestricted use, distribution, and reproduction in any medium, provided you give appropriate credit to the original author(s) and the source, provide a link to the Creative Commons license, and indicate if changes were made. The Creative Commons Public Domain Dedication waiver (http:// creativecommons.org/publicdomain/zero/1.0/) applies to the data made available in this article, unless otherwise stated.
IBD can cause immunosuppression in young chickens due to B lymphocyte depletion [4]. Furthermore, it has been reported that macrophages and monocytes may be susceptible to IBDV infection [4,5]. Macrophages have been thought to serve as IBDV carriers from the infection site in the gut to the bursa of Fabricius and other peripheral organs [5-7].

Immunosuppression decreases the resistance of these birds to other infections and also leads to an inadequate immune response to vaccination [8]. Immunosuppression following IBDV infection likely causes substantial economic loss due to vaccination failure, subsequent susceptibility to opportunistic pathogens, and loss of production [2]. The characteristic clinical signs of diseased chickens are dehydration and petechial hemorrhage at the thigh and breast muscles or at the junction between the proventriculus and the gizzard. The bursa of Fabricius of infected birds presents with inflammation, hemorrhage, or atrophy, 
depending on the infection period [9]. Vaccination plays an essential role in successfully controlling IBD $[10,11]$. At present, IBD vaccines are commercially available, including live attenuated, killed, immune complex, and vector vaccines [2]. Live attenuated vaccines, which are the most prevalently used IBD vaccines in the field, can be categorized into three groups: Mild, intermediate, and intermediate-plus or hot vaccines [12]. The viruses in mild vaccines exhibit poor efficacy in the presence of certain levels of maternally derived antibodies (MDAs) [13] and against very virulent IBDV (vvIBDV). In contrast, the intermediate and intermediate-plus or hot vaccines have much better efficacy and may break through the high level of MDAs and induce antibodies against IBDV. However, the various attenuation levels of commercially live IBD vaccines result in varying immunosuppression levels, increasing the birds' vulnerability to infection by other pathogens [14-16]. In addition, an efficient vaccination program depends on the time of vaccination, which can be affected by residual MDA levels [17]. Consequently, the safety and efficacy of these vaccine types still remain an essential concern. In commercial broiler breeder farms, killed IBDV vaccines have been routinely used to achieve a high antibody response against IBDV and consequently transmit the IBDV antibodies to the offspring, which may interfere with the immune response to vaccines $[17,18]$, an issue that farmers should be aware of before selecting an IBDV vaccine. In Thailand, similar to other countries, broiler producers tend to use the most virulent IBDV vaccines available, even if clinical Gumboro disease has not been commonly diagnosed in the previous broiler cycles.

This study aims to compare two commercially attenuated IBD vaccines in terms of safety, antibody response to the IBD vaccine, and immunosuppressive effect using the Newcastle disease virus (NDV) vaccination model in commercial broilers.

\section{Materials and Methods}

\section{Ethical approval}

The guidelines and legislative regulations on the use of animals for scientific purposes of Chulalongkorn University, Bangkok, Thailand, were followed as is certified in permission number 1931005.

\section{Study period and location}

This research was conducted for 9 months (January to September 2019), consisting of pre-research, experimental, and laboratory examination. The birds were reared in the experimental facilities, Faculty of Veterinary Science, Chulalongkorn University. The laboratory examinations were conducted at the Department of Veterinary Medicine, Chulalongkorn University.

\section{Animals}

A total of 216 female, unvaccinated, 1-day-old Cobb-500 broiler chicks were obtained from the same broiler breeder flock and hatchery. Breeder chickens were vaccinated with attenuated and inactivated IBDV vaccines as follows: At 14 days, Bursine 2 (Zoetis, USA); at 24 days, IBD Blen (Boehringer Ingelheim, Germany); and at 18 weeks, Provac 4 (Zoetis, USA). The chicks were maintained in three separate isolation units and fed ad libitum on commercial poultry feed (Betagro, Bangkok, Thailand).

\section{Experimental design}

A total of 216 female broilers were divided into three groups of 72 chicks each. Each group was divided into four replicates of 18 chicks each. In Group 1, broilers were vaccinated with intermediate-plus vaccine strain V217, one dose per bird orally administered at 15 days old, which is an optimal day for vaccination according to the Deventer formula. The breakthrough titer was 636 enzyme-linked immunosorbent assay (ELISA) units, with $75 \%$ of the flock being susceptible [19]. Each dose of vaccine contained approximately $10^{1.5}-10^{3} \mathrm{ELD}_{50}$ of IBDV. Seven days after IBDV vaccination, the chickens were vaccinated with one dose of the live ND vaccine (Hitchner B1 strain) by eye drop. In Group 2, broilers were vaccinated with the intermediate M.B. strain, one dose per bird orally administered at 15 days old, again according to the Deventer formula. The breakthrough titer was also 636 ELISA units with $75 \%$ of the flock being susceptible [19]. Seven days after IBD vaccination, the chickens were vaccinated with one dose of the live ND vaccine (Hitchner B1 strain) by eye drop. Group 3 was a negative control group, and the broilers did not receive any vaccine. At 15, 29, and 43 days old, all birds were weighed; feed intake was recorded, and feed conversion ratio (FCR) was calculated by the amount of feed consumed divided by the amount of weight gain in a period of time.

\section{Humoral immune response}

Specific antibody titers to IBDV and NDV were analyzed in serum samples using ELISA for IBDV strain D78 (BioChek, USA) and by a hemagglutination inhibition (HI) test, respectively. To collect hygienic samples, 20 birds per group were bled at 1 , 7, 15 (IBD vaccination day), 22, 29, 36, and 43 days old. A summary of sample collection in each group is presented in Table-1.

\section{Bursa:body weight (B:BW) ratio, B:BW index, and bursa scoring}

On vaccination day, and 7, 14, 21, and 28 days post-vaccination (dpv), one bird in each replicate was euthanized, and the bursa of Fabricius was collected to measure B:BW ratio (Table-1) and histopathological lesion score (HLS) determination. The B:BW ratio was calculated by the bursa of Fabricius weight $(\mathrm{g}) / \mathrm{BW}(\mathrm{g}) \times 1000$. The B:BW index was calculated by $\mathrm{B}: \mathrm{BW}$ ratio of vaccinated birds/B:BW ratio of the control group. HLS was determined according to Muskett et al. [20] using the following scale: (0) No damage; (1) mild necrosis in isolated follicles; (2) 
Table-1: Sample collections in each group.

\begin{tabular}{lcll}
\hline Sampling schedule & $\begin{array}{l}\text { Number of blood } \\
\text { samples/house }\end{array}$ & $\begin{array}{l}\text { Serological } \\
\text { analysis }\end{array}$ & $\begin{array}{l}\text { Number of bursa of } \\
\text { Fabricius }\end{array}$ \\
\hline 1 day of age & 20 & IBD, ND \\
7 days of age & 20 & IBD, ND \\
Vaccination day, 15 days of age & 20 & IBD, ND \\
7 dpv, 22 days of age & 20 & IBD, ND & 12 (4 from each group) \\
14 dpv, 29 days of age & 20 & IBD, ND & 12 (4 from each group) \\
21 dpv, 36 days of age & 20 & IBD, ND & 12 (4 from each group) \\
28 dpv, 43 days of age & 20 & IBD, ND & 12 \\
\hline
\end{tabular}

$\mathrm{dpv}=$ days post-vaccination, IBD=Infectious bursal disease, ND=Newcastle disease

moderate generalized lymphocyte depletion or isolated follicles, with severe depletion; (3) over $50 \%$ of follicles with severe lymphocyte depletion; (4) outline of follicles only remaining with few lymphocytes and increase in connective tissue, cysts, and thickened corrugated epithelium; and (5) loss of all follicular architecture with fibroplasia.

\section{RNA extraction and reverse transcription-poly-} merase chain reaction ( $R T-P C R$ )

At 43 days old, the bursae of Fabricius of six birds per group were collected, and RT-PCR was performed to detect IBDV. Furthermore, RNA virus was extracted from vaccines used in Groups 1 and 2. RNA from bursa tissue samples and vaccines was extracted using a commercial kit (NucleoSpin Extract Viral RNA Kit, Macherey-Nagel, Germany), as described by the manufacturer. The RT-PCR assay was conducted using a primer pair that amplifies a 743-bp region of VVP2 as previously described [21]. The RT-PCR products were analyzed using $1.2 \%$ agarose gel electrophoresis and visualized under an ultraviolet transilluminator.

\section{Nucleotide sequence analysis and phylogenetic tree construction}

The RT-PCR products of the vaccines from both groups were purified using a commercial kit (NucleoSpin Gel and PCR Clean-Up, MachereyNagel, Germany) and were submitted for nucleotide sequencing to First Base Laboratories (Seri Kembangan, Selangor, Malaysia) with specific primer sets. Basic Local Alignment Search Tool analysis was carried out on the website of the National Center for Biotechnology Information. Sequence analysis was performed using the MEGA 5.1 program [22]. The nucleotide sequences of two IBD vaccines used in this study, as well as very virulent, variant, classic, and serotype strains, were included. The genome sequences used as reference strains were taken from GenBank. The accession numbers included (1) very virulent strain: D49706 for OKYM, AF092943 for HK46, DQ916217 for Singapore97S182, AJ318897 for UK661, EF517528 for Harbin-1, DQ916248 for Thailand01_TH4, DQ916247 for Thailand01_TH3, GQ451330 for HLJ-0504, MW248904 for vaccine strain M.B., and AJ586962 for MB VP2, (2) variant strain: AF281238 for strain T1, DQ916213 for Mexico04M92, AF133904 for strain E, and DQ916181 for Colombia01C10, (3) classic strain: AF362747 for Cu-1wt, AJ586961 for Bursine plus, MW248903 for vaccine strain V217, DQ916252 for Thailand97 TH4, MW248905 for Thai4 Classic, AJ586966 for Nobilis 228E, and AY332560 for IBD Blen, and (4) Serotype 2: U30818 for OH, and AF362773 for 23/82. The phylogenetic tree was constructed using the Maximum Likelihood algorithm in the MEGA X program.

\section{Statistical analysis}

Antibody titers against IBDV and NDV, BW, and bursa weight were compared between groups using one-way analysis of variance and Duncan's multiple range tests. Differences between B:BW ratios and HLS were calculated using Chi-square and KruskalWallis tests. Differences between groups were considered significant at $p<0.05$. Statistical analysis was performed using Statistical Package for the Social Sciences for Windows v. 22 (IBM Corp., NY, USA).

\section{Results}

\section{Antibody titers against IBDV and NDV}

Maternal IBD antibody titers gradually decreased as broilers aged. At 1 and 7 days old, the broilers had not been vaccinated yet, so IBD and ND antibody titers were represented in all broiler groups. At 15 days old, the IBD vaccination date, there was no statistically significant difference in the IBD antibody titers of all groups. After vaccination, the IBD antibody titers in the vaccinated groups increased compared to the non-vaccinated group. At 36 and 43 days old, birds of Group 2 (the M.B. vaccinated group) displayed the highest IBD antibody titers (Group 1, $6099.1 \pm 4174.97$; and Group 2, $8677.95 \pm 3391.68$; $\mathrm{p}<0.05$ ) among all groups (Table-2). ND vaccines were administered at 22 days of age. At 36 and 43 days old, the ND antibody titers of birds in Group 1 displayed the highest ND antibody titers (30.85 31.74 and $21.85 \pm 28.94$, respectively) compared to Groups 2 and 3 (Table-3).

\section{BW and feed conversion ratio}

At 43 days old, broilers in Group 2 had the highest average BW $(1.94 \pm 0.41 \mathrm{~kg})$, while the FCR of broilers in Group 1 between 29 and 43 days was the lowest $(2.30 \pm 0.45)$ among the three experimental groups. However, differences in BW and FCR of broilers at 15, 29, and 43 days old were not statistically significant (Table-4). 


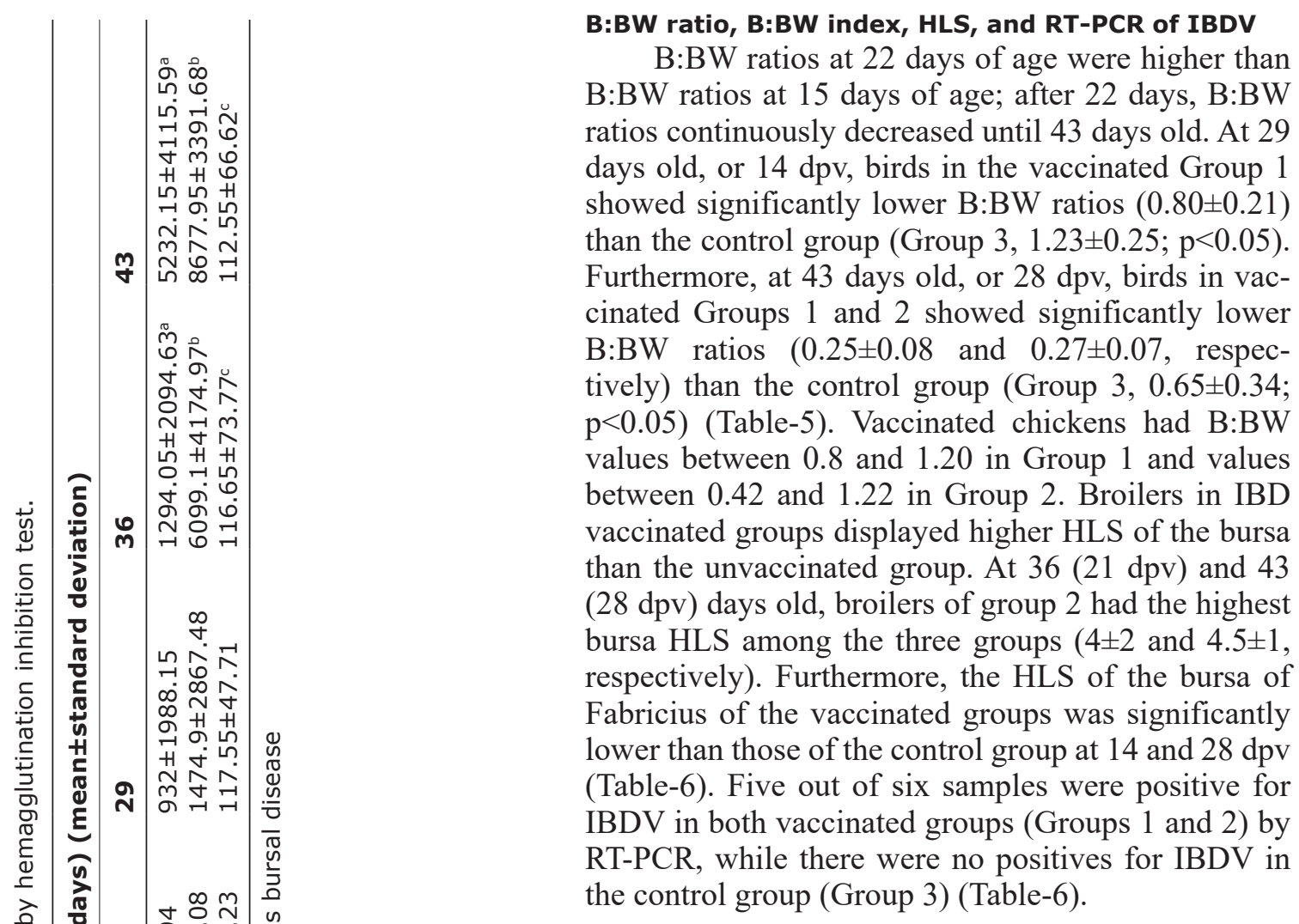

\section{Partial sequences of IBDV}

A partial sequence of the $V P 2$ indicated that virus from the vaccine strain V217 used in Group 1 was related to other classical IBDV strains, while the vaccine strain M.B. used in Group 2 was related to other vvIBDV strains. In addition, partial sequences of the VP2 from Groups 1 and 2 were $93.23 \%$ similar. The phylogenetic results are shown in Figure-1.

\section{Discussion}

IBD, also known as Gumboro disease, can cause morphologic and histological changes in the bursa of Fabricius. It can also cause significant economic losses due to the high mortality and high morbidity resulting from IBDV infection [2]. The immunosuppressive effect of IBDV vaccines is a concern due to the fact that the immunocompromised birds may not demonstrate sufficient titers of antibodies after vaccination against other diseases, such as NDV [4]. The IBDV vaccination has been used in the chicken industry worldwide to prevent IBDV infection. In Thailand, broiler breeders, broilers, and layers have been commonly vaccinated with the intermediate and/or intermediate-plus strains of IBD live and/or killed vaccines, depending on the type of chickens. Long-lived birds are also routinely vaccinated with killed IBDV vaccines to maintain a certain level of immune response to IBDV for prolonged protection. In this study, we compared two attenuated IBD vaccines (intermediate and intermediate-plus strains) in terms of safety, antibody response to IBD vaccine, and immunosuppressive effect using the NDV vaccination model in commercial broilers. 
Table-3: Antibody titers against ND by hemagglutination inhibition test.

\begin{tabular}{lccccccc}
\hline Group & \multicolumn{6}{c}{ ND antibody titers at different ages (days) (mean \pm standard deviation) } \\
\cline { 2 - 8 } & $\mathbf{1}$ & $\mathbf{7}$ & $\mathbf{1 5}$ & $\mathbf{2 2}$ & $\mathbf{2 9}$ & $\mathbf{3 6}$ & $\mathbf{4 3}$ \\
\hline 1 (V217) & & & $2.95 \pm 1.67$ & $2.00 \pm 1.12$ & $1.15 \pm 0.67$ & $30.85 \pm 31.74^{\mathrm{a}}$ & $21.85 \pm 28.94^{\mathrm{a}}$ \\
2 (M.B.) & $124.80 \pm 83.64$ & $16.40 \pm 6.07$ & $3.30 \pm 1.49$ & $2.05 \pm 0.94$ & $1.10 \pm 0.31$ & $15.55 \pm 9.84^{\mathrm{b}}$ & $17.30 \pm 15.63^{\mathrm{a}, \mathrm{b}}$ \\
3 (control) & & & $3.20 \pm 1.51$ & $2.50 \pm 1.32$ & $1.20 \pm 0.41$ & $1.00 \pm 0.00^{\mathrm{c}}$ & $1.00 \pm 0.00^{\mathrm{c}}$ \\
\hline
\end{tabular}

Different superscripts in each column mean statistically significant difference $(p<0.05)$. ND=Newcastle disease

Table-4: Body weight (kilogram) and FCR.

\begin{tabular}{|c|c|c|c|c|c|c|}
\hline \multirow[t]{2}{*}{ Group } & \multicolumn{3}{|c|}{ Body weight (mean \pm SD) } & \multicolumn{3}{|c|}{ FCR (mean \pm SD) } \\
\hline & 15 day & 29 day & 43 day & 1-15 day & 15-29 day & 29-43 day \\
\hline 1 (V217) & $0.56 \pm 0.04$ & $1.39 \pm 0.16$ & $1.86 \pm 0.45$ & $1.20 \pm 0.03$ & $0.89 \pm 0.06$ & $2.30 \pm 0.45$ \\
\hline 2 (M.B.) & $0.56 \pm 0.03$ & $1.43 \pm 0.09$ & $1.94 \pm 0.41$ & $1.17 \pm 0.03$ & $0.94 \pm 0.00$ & $2.40 \pm 0.2$ \\
\hline 3 (control) & $0.57 \pm 0.06$ & $1.43 \pm 0.07$ & $1.93 \pm 0.41$ & $1.17 \pm 0.01$ & $0.93 \pm 0.04$ & $2.36 \pm 0.48$ \\
\hline
\end{tabular}

FCR $=$ Feed conversion ratio, $\mathrm{SD}=$ Standard deviation

Table-5: Bursa weight (gram), BW (gram), and B:BW ratios.

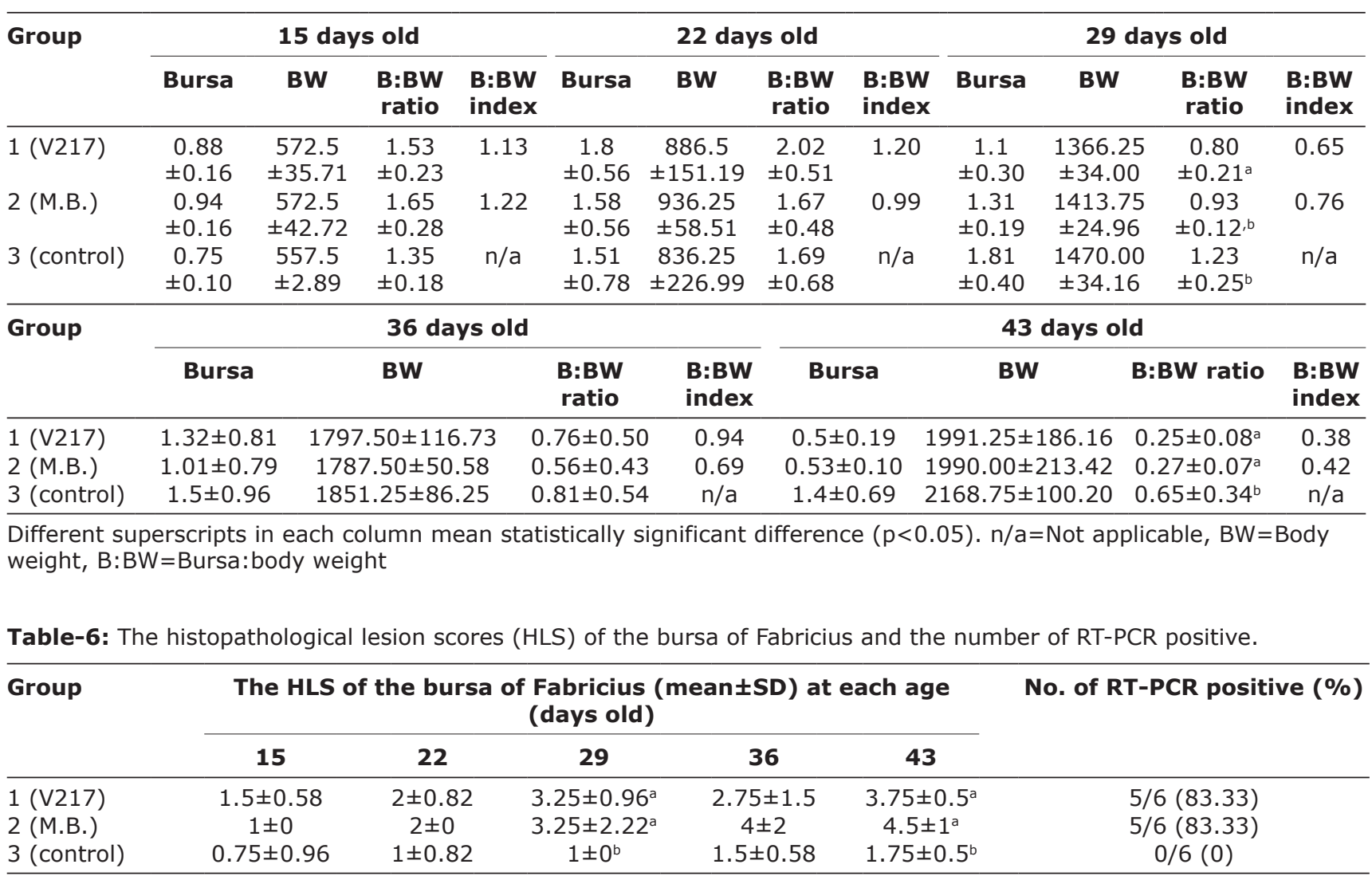

Different superscripts in each column mean statistically significant difference $(p<0.05)$

One-day-old chicks in both treatment groups came from the same hatchery and the same breed, and we randomly sampled their blood checking for IBDV antibody levels. Before each trial, a date for the IBD vaccination was calculated according to the Deventer formula [19] to reduce factors that may have influenced the results of the study. Interestingly, our study demonstrated that, although the IBD vaccine M.B. strain is commercially registered as an intermediate type, it was found that the M.B. vaccinated birds displayed significantly higher IBD antibody titers than the V217 vaccinated birds, which is rated as an intermediate-plus strain, at 14 and $21 \mathrm{dpv}$. Furthermore, the M.B.-vaccinated birds demonstrated significantly lower ND antibody titer by HI test at $14 \mathrm{dpv}$.

Rautenschlein et al. [23] reported that the intermediate-plus IBDV vaccine increased transient suppression of NDV antibody titer after NDV vaccination in commercial broilers, whereas a permanent suppression of ND antibody titer was observed in SPF layers. Although IBD is of great economic significance in the broiler industry and many studies have been conducted to determine the efficacy and immunosuppressive effects in SPF chickens [14,24-26], few studies have been performed in commercial broilers with residual MDAs [23,27-29]. 


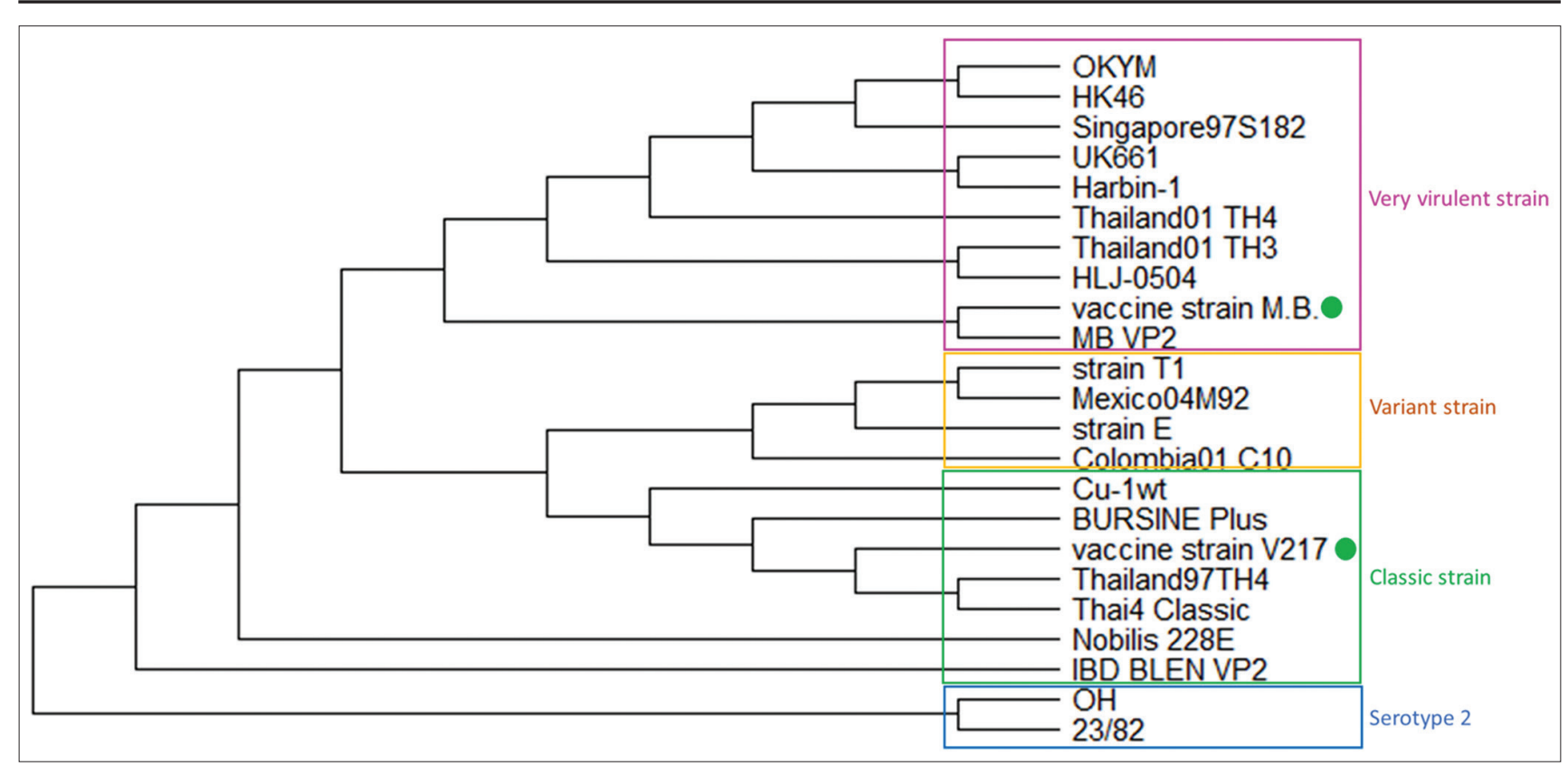

Figure-1: Phylogenetic analysis revealed that M.B. strain belongs to very virulent infectious bursal disease virus (IBDV) and V217 belongs to classic IBDV strain.

Before IBDV live vaccines are put on the markets, most IBDV vaccines are evaluated for their immunosuppressive effects in SPF layer-type chickens to categorize the IBD vaccine as mild, intermediate, or intermediate-plus type $[25,26]$, which may cause different results when compared to IBDV-vaccinated commercial broilers with residual MDAs. In addition, Lazarus et al. [30] described that the IBDV M.B. live vaccine is an intermediate to intermediate-plus strain originating from a vvIBDV, in agreement with our phylogenetic results, which showed that the IBDV vaccine M.B. strain was grouped in a cluster of vVIBDV, while the IBDV vaccine strain V217 was grouped in a cluster of classical strains of IBDV. This may be the reason why M.B. vaccinated birds showed a higher IBD antibody titer and greater immunosuppressive effect than the group that received the V217 strain.

BW and FCR of both treatment and control groups were not statistically significantly different. We found that the B:BW ratio of both treatment groups was markedly decreased at $14 \mathrm{dpv}$, and $\mathrm{B}: \mathrm{BW}$ of treatment groups was significantly lower than that of the control group, similar to a previous study [31]. Likewise, the HLS of the bursa of Fabricius of treatment groups was significantly lower than that of the control group at 14 and $28 \mathrm{dpv}$.

To confirm that the IBDV vaccine in both groups could invade the bursal follicles, RT-PCR was performed to verify whether the follicles were positive for IBDV. The results indicated that $83.33 \%$ of the tested bursae were positive for IBDV, indicating that the broilers received proper IBDV vaccination in both treatment groups. In this study, we found that both IBDV vaccines could be present in the bursae of Fabricius at $28 \mathrm{dpv}$, which is in agreement with the study of Iván et al. [32], so broiler farmers may be made aware that IBDV can be detected as a result of vaccination.

\section{Conclusion}

This study was conducted to compare two attenuated IBD vaccines (strain V217 and strain M.B.) in terms of safety, antibody response to IBD vaccine, and antibody response to the Newcastle disease vaccine in commercial broilers. The V217-vaccinated group presented the highest NDV humoral response from 7 days dpv to the end of the study. The mean NDV antibody titer of the V217 group was significantly higher than the M.B.-vaccinated group at 14 dpv $(p<0.05)$. In addition, the V217 strain-induced lower bursal lesions post-IBD vaccination and higher $\mathrm{B}: \mathrm{BW}$ ratios at 7 and $21 \mathrm{dpv}$ compared to the M.B. group. The higher B:BW ratio, lower bursal lesions, and higher ND seroconversion present in the V217 group indicated that the V217 strain-induced lower immunosuppressive effects compared to the M.B strain. The results of this study indicate that selecting IBD vaccine merits consideration, avoiding immunosuppressive effects induced by live IBD vaccination and the impact on the response to subsequent vaccines is important.

\section{Authors' Contributions}

TT: Conceptualization, methodology, investigation, data curation, writing - original draft and visualization, NC: Methodology, software and formal analysis, NCh: Conceptualization, methodology, investigation, resources, writing - review and editing, supervision, funding acquisition, and corresponding author. All authors read and approved the final manuscript. 


\section{Acknowledgments}

This research was funded by Elanco Animal Health (Spain) Limited, (Grant numberRES_62_131_31_015). We are grateful to the staff of Avian Health Research Unit, Chulalongkorn University, Thailand, for all support.

\section{Competing Interests}

No person from Elanco Animal Health was involved at any stage of the study.

\section{Publisher's Note}

Veterinary World remains neutral with regard to jurisdictional claims in published institutional affiliation.

\section{References}

1. Aliyu, H.B., Sa'idu, L., Jamilu, A., Andamin, A.D. and Akpavie, S.O. (2016) Outbreaks of virulent infectious bursal disease in flocks of battery cage brooding system of commercial chickens. J. Vet. Med., 2016: 1-7.

2. Eterradossi, Y.M.S. (2020) Infectious bursal disease. In: Swayne, D.E., editors. Diseases of Poultry. $14^{\text {th }}$ ed. Wiley Blackwell, New York. p257-283.

3. Michel, L.O. and Jackwood, D.J. (2017) Classification of infectious bursal disease virus into genogroups. Arch. Virol., 162(12): 3661-3670.

4. Spackman, E., Stephens, C.B. and Pantin-Jackwood, M.J. (2018) The effect of infectious bursal disease virus-induced immunosuppression on vaccination against highly pathogenic avian influenza virus. Avian Dis., 62(1): 36-44.

5. Liu, A., Li, H., Qi, X., Wang, Q., Yang, B., Wu, T., Yan, N., Li, Y., Pan, Q. and Gao, Y. (2019) Macrophage migration inhibitory factor triggers inflammatory responses during very virulent infectious bursal disease virus infection. Front. Microbiol., 10: 1-15.

6. Rasoli, M., Yeap, S.K., Tan, S.W., Roohani, K., KristeenTeo, Y.W., Alitheen, N.B., Abd Rahaman, Y., Aini, I., Bejo, M.H. and Kaiser, P. (2015) Differential modulation of immune response and cytokine profiles in the bursae and spleen of chickens infected with very virulent infectious bursal disease virus. BMC Vet. Res., 11(1): 75.

7. Lee, C.C., Wu, C.C. and Lin, T.L. (2015) Role of chicken melanoma differentiation-associated gene 5 in induction and activation of innate and adaptive immune responses to infectious bursal disease virus in cultured macrophages. Arch. Virol., 160(12): 3021-3035.

8. Orakpoghenor, O., Oladele, S.B. and Abdu, P.A. (2020) Infectious bursal disease: Transmission, pathogenesis, pathology and control-an overview. World Poult. Sci. J., 76(2): $1-12$.

9. Chansiripornchai, N. and Sasipreeyajan, J. (2009) Comparison of the efficacy of the immune complex and conventionally live vaccine in broilers against infectious bursal disease infection. Thai J. Vet. Med., 39(2): 115-120.

10. Rautenschlein, S., Kraemer, C., Vanmarcke, J. and Montiel, E. (2005) Protective efficacy of intermediate and intermediate plus infectious bursal disease virus (IBDV) vaccines against very virulent IBDV in commercial broilers. Avian Dis., 49(2): 231-237.

11. Alkie, T.N. and Rautenschlein, S. (2016) Infectious bursal disease virus in poultry: Current status and future prospects. Vet. Med., 7: 9-18.

12. Olesen, L., Dijkman, R., Koopman, R., van Leeuwen, R., Gardin, Y., Dwars, R., de Bruijn, N., Boelm, G., Elattrache, J. and de Wit, J. (2018) Field and laboratory findings following the large-scale use of intermediate type infectious bursal disease vaccines in Denmark. Avian Pathol., 47(6):
595-606.

13. Naqi, S.A., Marquez, B. and Sahin, N. (1983) Maternal antibody and its effect on infectious bursal disease immunization. Avian Dis., 27(3): 623-631.

14. Rautenschlein, S., Yeh, H.Y. and Sharma, J.M. (2003) Comparative immunopathogenesis of mild, intermediate, and virulent strains of classic infectious bursal disease virus. Avian Dis., 47(1): 66-78.

15. Tomás, G., Marandino, A., Courtillon, C., Amelot, M., Keita, A., Pikula, A., Hernández, M., Hernández, D., Vagnozzi, A. and Panzera, Y. (2019) Antigenicity, pathogenicity and immunosuppressive effect caused by a South American isolate of infectious bursal disease virus belonging to the "distinct" genetic lineage. Avian Pathol., 48(3): 245-254.

16. Kurukulasuriya, S., Ahmed, K.A., Ojkic, D., Gunawardana, T., Goonewardene, K., Gupta, A., ChowLockerbie, B., Popowich, S., Willson, P. and Tikoo, S.K. (2017) Modified live infectious bursal disease virus (IBDV) vaccine delays infection of neonatal broiler chickens with variant IBDV compared to turkey herpesvirus (HVT)IBDV vectored vaccine. Vaccine, 35(6): 882-888.

17. Jackwood, D.J. (2017) Advances in vaccine research against economically important viral diseases of food animals: Infectious bursal disease virus. Vet. Microbiol., 206: 121-125.

18. Zhou, X., Wang, D., Xiong, J., Zhang, P., Li, Y. and She, R. (2010) Protection of chickens, with or without maternal antibodies, against IBDV infection by a recombinant IBDV-VP2 protein. Vaccine, 28(23): 3990-3996.

19. de Wit, J. (1998) Gumboro disease: Estimation of optimal time of vaccination by the deventer formula. Vol. 3. In: Proceedings of the $3^{\text {rd }}$ Meeting Working Group. p19-22.

20. Muskett, J., Hopkins, I., Edwards, K. and Thornton, D. (1979) Comparison of two infectious bursal disease vaccine strains: Efficacy and potential hazards in susceptible and maternally immune birds. Vet. Rec., 104(15): 332-334.

21. Jackwood, D.J. and Sommer-Wagner, S. (2007) Genetic characteristics of infectious bursal disease viruses from four continents. Virology, 365(2): 369-375.

22. Tamura, K., Peterson, D., Peterson, N., Stecher, G., Nei, M. and Kumar, S. (2011) MEGA5: Molecular evolutionary genetics analysis using maximum likelihood, evolutionary distance, and maximum parsimony methods. Mol. Biol. Evol., 28(10): 2731-2739.

23. Rautenschlein, S., Kraemer, C., Montiel, E., Vanmarcke, J. and Haase, C. (2007) Bilateral effects of vaccination against infectious bursal disease and Newcastle disease in specific-pathogen-free layers and commercial broiler chickens. Avian Dis., 51(1): 14-20.

24. Mazariegos, L.A., Lukert, P.D. and Brown, J. (1990) Pathogenicity and immunosuppressive properties of infectious bursal disease intermediate strains. Avian Dis., 34(1): 203-208

25. Giambrone, J.J. and Clay, R.P. (1986) Evaluation of the immunogenicity, stability, pathogenicity, and immunodepressive potential of four commercial live infectious bursal disease vaccines. Poult. Sci., 65(7): 1287-1290.

26. Giambrone, J.J. and Clay, R.P. (1986) Efficacy of coarse spray administration of commercial intermediate infectious bursal disease vaccines. Poult. Sci., 65(4): 807-808.

27. Giambrone, J.J., Dormitorio, T. and Brown, T. (2001) Safety and efficacy of in ovo administration of infectious bursal disease viral vaccines. Avian Dis., 45(1): 144-148.

28. Alam, J., Rahman, M.M., Sil, B.K., Khan, M.S.R., Giasuddin, M. and Sarker, M.S.K. (2002) Effect of maternally derived antibody on vaccination against infectious bursal disease (Gumboro) with live vaccine in broiler. Int J. Poult. Sci., 1(4): 98-101.

29. Giambrone, J.J. and Closser, J. (1990) Efficacy of live vaccines against serologic subtypes of infectious bursal disease virus. Avian Dis., 34(1): 7-11. 
30. Lazarus, D., Pasmanik-Chor, M., Gutter, B., Gallili, G., Barbakov, M., Krispel, S. and Pitcovski, J. (2008) Attenuation of very virulent infectious bursal disease virus and comparison of full sequences of virulent and attenuated strains. Avian Pathol., 37(2): 151-159.

31. Ashash, U., Noach, C., Perelman, B., Costello, C., Sansalone, P., Brazil, T. and Raviv, Z. (2019) In ovo and day of hatch application of a live infectious bursal disease virus vaccine to commercial broilers. Avian Dis., 63(4): 713-720.

32. Iván, J., Velhner, M., Ursu, K., Germán, P., Mató, T., Drén, C.N. and Mészáros, J. (2005) Delayed vaccine virus replication in chickens vaccinated subcutaneously with an immune complex infectious bursal disease vaccine: Quantification of vaccine virus by real-time polymerase chain reaction. Can. J. Vet. Res., 69(2): 135-142.

$* * * * * * * *$ 